\title{
Understanding disease mechanisms at the nanoscale: endothelial apoptosis and microparticles in COPD
}

\author{
Tom Wilkinson ${ }^{1,2}$
}

The clinical burden of COPD remains a global health challenge that is not being met by current preventive and therapeutic strategies. ${ }^{1}$ For decades, the key features of this heterogeneous condition have been studied and the associations between airways disease, emphysema, systemic illness and tobacco smoking have been explored. Despite significant advances in our understanding of the aetiology of important clinical aspects of disease including the heterogeneity of airway inflammation ${ }^{2}$ and the pathogenesis of exacerbations, ${ }^{3}$ there remain significant gaps in our understanding of the mechanisms underlying key facets of the condition including the origins of emphysema.

A number of potential mechanisms of disease have been proposed to explain the progressive loss of lung parenchyma in response to tobacco and biomass smoke exposure. ${ }^{4}$ Focus on protease imbalance and neutrophilic inflammation ${ }^{5}$ on one hand and T-cell-mediated pathology on another ${ }^{6}$ have offered some insights into contributory mechanisms but to date have failed to offer a route to disease modifying therapy or even convincing biomarkers of disease progression.

Furthermore, it is increasingly apparent that COPD is both a pulmonary and systemic condition, with a significant contribution to the associated morbidity and mortality driven by an increased incidence and severity of cardiovascular disease. ${ }^{7}$ It has been recognised that endothelial pathology is a key component of COPD, both in the manifestation of a systemic disease process and in the development of discrete lung pathology particularly emphysema. ${ }^{8}$

The pulmonary vasculature and its role in COPD have been somewhat of a 'black box'-implicated as a key factor in disease

${ }^{1}$ NIHR Southampton Respiratory Biomedical Research Unit, University Hospital Southampton NHS Foundation Trust, Southampton, UK; ${ }^{2}$ Academic Unit of Clinical and Experimental Sciences, Faculty of Medicine, University of Southampton, Southampton, UK

Correspondence to Professor Tom Wilkinson, Academic Unit of Clinical and Experimental Sciences, Faculty of Medicine, University of Southampton, Mailpoint 810, UHS, Southampton SO166YD, UK; t.wilkinson@soton.ac.uk but difficult to study in vivo and with no accessible biomarker of relevant pathology available. The endothelium is a vast monolayer of endothelial cells which forms the internal lining of all blood vessels including those in the lung. It has an established role in the control of blood flow and is increasingly recognised as a major contributor in the control of inflammation, immune responses and disease in all organs. ${ }^{9}$ Cigarette smoking has been identified as a major driver to endothelial dysfunction and has been implicated as a causal factor in endothelial apoptosis. Key to developing our understanding of endothelial pathology in the lung has been the previous work by Gordon et $a l^{8}$ that microparticles were identified as elevated in smokers and were associated with a reduction in pulmonary diffusion capacity.

Microparticles are cell membrane derived vesicles that are produced by many different cell types in response to changes in activation status, stress or injury. Microparticles constitute the larger end of of spectrum of subcellular particles termed extracellular vesicles (EVs) which range from exosomes at $30-90 \mathrm{~nm}$ to microparticles at $100-1000 \mathrm{~nm} .{ }^{10}$ Interest in this field is great because their presence at elevated levels in the blood has been associated with a range of conditions which are known to impact on the endothelium including ischaemic heart disease, ${ }^{11}$ stroke $^{12}$ and even obstructive sleep apnoea, ${ }^{13}$ and also unlike protein biomarkers, microparticles and other subcellular vesicles can be characterised by markers which offer insights into their cellular origins and even the biological processes key to their production. In addition, the biology of EV opens the door to understanding how cell-to-cell communication can act at distance and how disease processes active in one organ may influence distant organ or systemic health.

In a study reported in Thorax, the authors present an important finding that markers of pulmonary endothelial apoptosis namely microparticles are elevated in COPD and persist despite smoking cessation. ${ }^{14}$ A cohort of well-phenotyped subjects with COPD was compared with non-smokers and smoking controls. The key finding was that levels of endothelial microparticles were significantly elevated in smoking controls and patients with COPD and that interestingly levels remained elevated in subjects with COPD despite smoking cessation but returned to normal in subjects without COPD who quit smoking. Of note these levels did not relate to measures of lung function or inhaled therapies suggesting a parallel component of disease to those currently assessed and treated.

These data are enticing on many levels. First, it is refreshing to see a clinical study using a 'natural experimental design' benefitting from the follow-up of subjects who were able to stop smoking. Animal models of smoking have been established but their relevance to the pathogenesis of COPD remains questionable and so these in vivo human data are particularly compelling. Second, the suggestion that a measurable marker of pulmonary vascular pathology is available which is accessible via peripheral blood sampling is exciting. To understand the biology of microparticles, their relevance to the lung endothelium and mechanisms behind their production, a detailed phenotyping protocol was used. Peripheral blood microparticles $<1.5 \mu \mathrm{m}$ were characterised by flow cytometry. The validated endothelial markers platelet endothelial cell adhesion molecule (PECAM) (CD31) and E-selectin (CD62E) were used to confirm the cellular origin of the particles and the likely mechanism-apoptosis versus cellular activation-of their production. To ascertain the organ of origin of the particles sampled from the peripheral blood, staining for ACE which is strongly and constitutively expressed by the pulmonary endothelium, was employed. This offered unique insights into the biology of this poorly understood compartment.

However, many questions remain and the relevance of this signal to conventional measures of disease activity or progression is yet to be explored. Such a well-defined marker may provide a unique opportunity to study the natural history of pulmonary endothelial disease for the first time, appears responsive to relevant exposures in vivo and may help characterise a new phenotype of patients with COPD with active vascular-mediated disease. Measurement of specific microparticles or even estimation of PECAM levels in the peripheral blood therefor requires exploration in larger longitudinal studies.

The hunt for relevant biomarkers of disease progression in COPD has been a long one and to date has focused on traditional models of airway inflammation 
and quantification of cellular or protein targets. ${ }^{15}$ Due to the heterogeneity of the condition and the difficulty in obtaining adequate samples which reflect the relevant pathology of the lung, the task is still a work in progress. The emerging field of subcellular particles is a fast moving one, recognising that careful phenotyping of these nanoscale targets can offer insights into hard to reach cell's function should encourage more work to develop and hopefully more disease understanding will follow. The potential utility of such a marker will of course increase as our understanding of the mechanisms driving emphysema progression develops and new targets are therapeutically identified.

To impact on the enormous clinical burden driven by COPD, an improved understanding of all the contributory aspects of this heterogeneous disease will be key. Alongside new mechanistic insights, relevant biomarkers are still needed to further develop a strategy to stratify patients to better predict risk and response to specific therapies. This important study of microparticles takes the field a small but significant step forward on this journey and raises the profile of an important disease endotype of COPD driven by microvascular pathology.

Competing interests None declared.
Provenance and peer review Commissioned; externally peer reviewed.

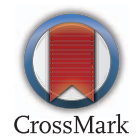

To cite Wilkinson T. Thorax 2016;71:1078-1079.

Published Online First 26 September 2016

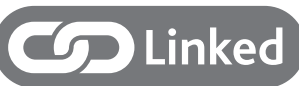

http://dx.doi.org/10.1136/thoraxjnl-2015-208274

Thorax 2016;71:1078-1079.

doi:10.1136/thoraxjnl-2016-208993

\section{REFERENCES}

1 Global Initiative for Chronic Obstructive Lung Disease. 2011. http://www.goldcopd.com/ (last accessed 7 Jul 2016).

2 Barnes PJ. Inflammatory mechanisms in patients with chronic obstructive pulmonary disease. $J$ Allergy Clin Immunol 2016;138:16-27.

3 Wilkinson TM, Hurst JR, Perera WR, et al. Effect of interactions between lower airway bacterial and rhinoviral infection in exacerbations of COPD. Chest 2006;129:317-24.

4 Taraseviciene-Stewart L, Voelkel NF. Molecular pathogenesis of emphysema. I Clin Invest 2008;118:394-402.

5 Ostridge K, Williams N, Kim V, et al. Relationship between pulmonary matrix metalloproteinases and quantitative $\mathrm{CT}$ markers of small airways disease and emphysema in COPD. Thorax 2016;71:126-32.
6 McKendry RT, Spalluto CM, Burke $\mathrm{H}$, et al. Dysregulation of antiviral function of CD8(+) T cells in the chronic obstructive pulmonary disease lung. Role of the PD-1-PD-L1 axis. Am J Respir Crit Care Med 2016;193:642-51.

7 Maclay JD, MacNee W. Cardiovascular disease in COPD: mechanisms. Chest 2013;143:798-807.

8 Gordon C, Gudi K, Krause A, et al. Circulating endothelial microparticles as a measure of early lung destruction in cigarette smokers. Am J Respir Crit Care Med 2011;184:224-32.

9 Rajendran P, Rengarajan T, Thangavel J, et al. The vascular endothelium and human diseases. Int I Biol Sci 2013;9:1057-69.

10 EL Andaloussi S, Mäger I, Breakefield XO, et al. Extracellular vesicles: biology and emerging therapeutic opportunities. Nat Rev Drug Discov 2013;12:347-57.

11 Mallat Z, Benamer H, Hugel B, et al. Elevated levels of shed membrane microparticles with procoagulant potential in the peripheral circulating blood of patients with acute coronary syndromes. Circulation 2000;101:841-3.

12 Simak J, Gelderman MP, Yu H, et al. Circulating endothelial microparticles in acute ischemic stroke: a link to severity, lesion volume and outcome. J Thromb Haemost 2006;4: 1296-302.

13 Ayers L, Ferry B, Craig S, et al. Circulating cell-derived microparticles in patients with minimally symptomatic obstructive sleep apnoea. Eur Respir J 2009:33:574-80.

14 Strulovici-Barel Y, Staudt MR, Krause A, et al. Persistence of circulating endothelial microparticles in COPD despite smoking cessation. Thorax 2016;71: 1137-44.

15 Thomsen $\mathrm{M}$, Ingebrigtsen TS, Marott JL, et al. Inflammatory biomarkers and exacerbations in chronic obstructive pulmonary disease. JAMA 2013;309:2353-61. 